\title{
Performed Study Subject Milestone Registration Date
}

National Cancer Institute

\section{Source}

National Cancer Institute. Performed Study Subject Milestone Registration Date. NCI

Thesaurus. Code C93967.

The date (and time) the subject has been registered to the study assuming they have finished screening and have been found eligible. 